\title{
3D DESIGN OF THE FUSION-FEM DEPRESSED COLLECTOR USING THE GENERAL PARTICLE TRACER (GPT) CODE.
}

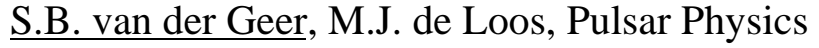 \\ W.H. Urbanus, A.G.A. Verhoeven, FOM-Rijnhuizen, Association EURATOM-FOM
}

\section{Abstract}

The "Rijnhuizen" Fusion Free-Electron Maser (FEM) is the pilot experiment for a high power, mm-wave source, tunable in the range 130-260 GHz. The FEM has generated $730 \mathrm{~kW}$ output power during $10 \mu \mathrm{s}$ pulses.

To increase the overall efficiency to over $50 \%$ and to reach a pulse length of at least $100 \mathrm{~ms}$, an electron beam charge and energy recovery system is currently being designed and installed. This system consists of an electrostatic decelerator, which decels the beam from $2 \mathrm{MeV}$ to an average of $200 \mathrm{keV}$, and a depressed collector. The EM-wave interaction inside the undulator can result in an energy spread of $300 \mathrm{keV}$ behind the decelerator.

The multi-stage collector is designed so that electrons fall on the backside of one of three electrodes, thus ensuring that secondary particles will immediately be accelerated back towards the electrodes. However, scattered primary electrons can cause back streaming, hereby reducing the efficiency and possibly damaging the machine.

To reduce this back streaming to below a tolerable $0.1 \%$, the General Particle Tracer (GPT) code is being used to calculate primary and scattered particle trajectories inside the collector. It will be shown that an off-axis bending scheme, using a rotating perpendicular magnetic field lowers the back streaming and hereby increases the pulse length of the machine. The bending scheme also improves the power dissipation in the collector.

\section{INTRODUCTION}

The Fusion-FEM is the prototype of a high power, electrostatic mm-wave source, tunable in the range 130$260 \mathrm{GHz}$ [1]. The device is driven by a $2 \mathrm{MeV}, 12 \mathrm{~A} \mathrm{dc}$ electron beam and is designed to generate $1 \mathrm{MW}$ microwave power, see Table A. Without decelerator and collector, the FEM has produced $730 \mathrm{~kW}$ during $10 \mu \mathrm{s}$ pulses. In the future FEMs can be used as power sources for electron cyclotron applications on magnetically confined plasmas in fusion research devices.

Because of its flexibility and capabilities, the General Particle Tracer (GPT) code has been used for the complete design of the FEM beam line [2]. The simulations include FEL interaction, space-charge effects, imported electrostatic and magnetostatic field data and misalignment.

To be able to meet the target efficiency of over $50 \%$ and a pulse length of $100 \mathrm{~ms}$, a beam and energy recovery system is crucial. The GPT code has been used to design this system and investigate the effects of an off-axis bending scheme. In this paper we will present simulation results for the FEM depressed collector.

Table A: Principal design parameters of the Fusion FEM

\begin{tabular}{ll}
\hline Parameter & Value \\
\hline Electron beam current & $12 \mathrm{~A}$ \\
Electron beam energy & $1.35-2 \mathrm{MeV}$ \\
Pulse length & $100 \mathrm{~ms}$ \\
Microwave frequency & $130-260 \mathrm{GHz}$ \\
Microwave net power & $1 \mathrm{MW}$ \\
Target system efficiency & $\geq 50 \%$ \\
Target current losses & $\leq 20 \mathrm{~mA}$ \\
Linear gain per pass & $7-10$ \\
Gain at saturation & 3.5 \\
Waveguide parameters & $\mathrm{HE}$ in $15 \times 20 \mathrm{~mm}^{2}$ \\
Undulator period & $40 \mathrm{~mm}$ \\
First undulator section & 20 periods with $0.20 \mathrm{~T}$ \\
Second undulator section & 14 periods with $0.16 \mathrm{~T}$ \\
\hline
\end{tabular}

\section{BEAM AND ENERGY RECOVERY}

The beam and energy recovery system of the Fusion FEM consists of an electrostatic decelerator, a transport line and a depressed collector. GPT simulations have shown that the EM-wave interaction in the undulator results in a maximum energy spread of $300 \mathrm{keV}$ behind the decelerator. For this energy distribution a depressed collector with three electrodes will suffice to obtain the required efficiency.

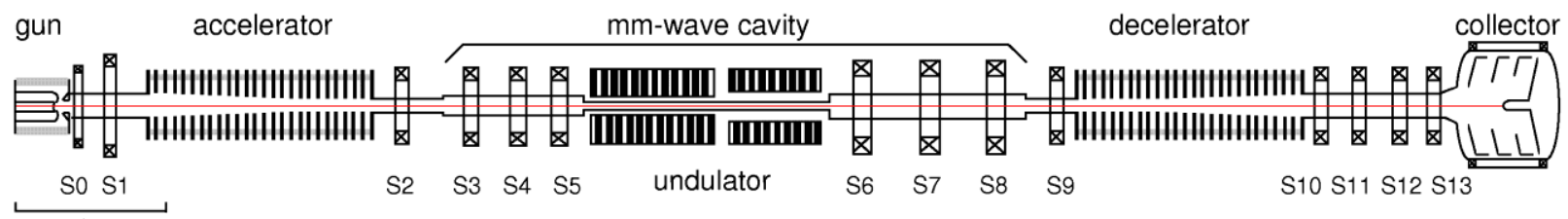

Figure 1: FEM beam line. 


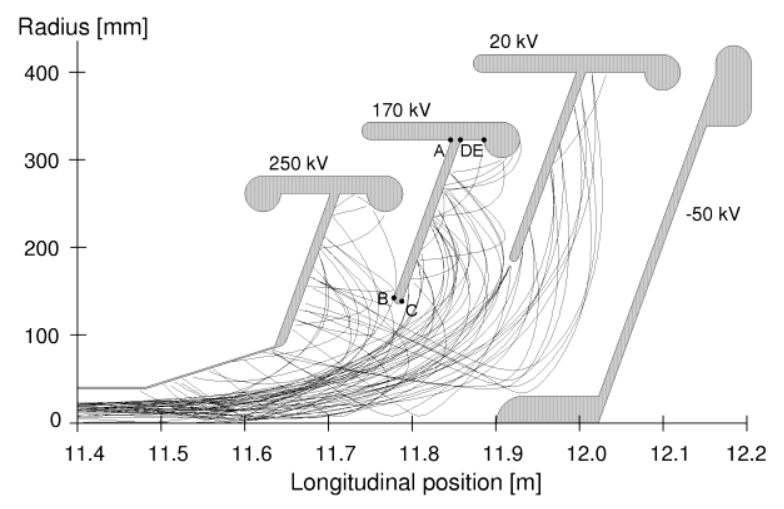

Figure 2: FEM depressed collector with sample trajectories. Both primary and scattered particles are shown.

As shown in Figure 2, in the depressed collector the electrons are collected on the backside of the electrodes. This assures secondary electrons to fall back on the electrodes. Scattered electrons, however, have an energy close to the initial energy and can cause back streaming via multiple scattering. As all beam loss and back streaming current has to be delivered by the $2 \mathrm{MV}$ power supply, which can deliver only $20 \mathrm{~mA}$, at least $99.8 \%$ of the beam needs to be collected. Furthermore, back streaming could damage the machine.

To decrease back streaming, an off-axis deflection system is being installed. It consists of four solenoids generating a rotating perpendicular magnetic field. The coils are mounted directly on the surface of the collector housing to increase the effective area, resulting in a bent elliptical shape.

The deflection system causes an angle dependency for the current distribution on the collector electrodes, shown as an example in Figure 3. Only the top-bottom coils are powered, but the beam is pushed vertically due to the tail field of S13.
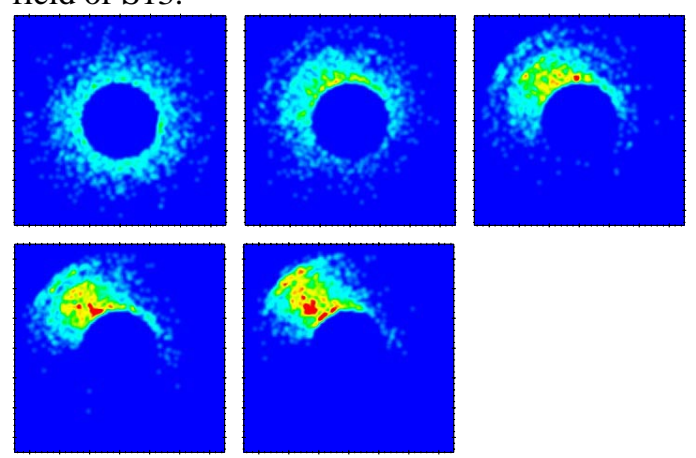

Figure 3: Simulation results for the current dissipation on the second collector plate. The plots represent $0,0.5,1.0$, 1.5 and $2.0 \mathrm{mT}$ in the deflection system.

\section{GPT}

The General Particle Tracer (GPT) [3,4] is a software package developed to aid in the design of accelerators and beam lines by using modern, particle tracking techniques. GPT is capable of tracking any number of particles through complex electromagnetic fields taking all 3D effects and space-charge forces into account. A $5^{\text {th }}$ order embedded Runge-Kutta integrator with adaptive stepsize control produces highly accurate and reliable results, while the simulation time is kept to a minimum.

The fields of physical structures are represented by so called elements. GPT comes with a large set of standard elements for basic structures such as solenoids, quadrupoles and accelerating structures. However, for specific cases custom elements can easily be added. Elements can be positioned anywhere and in any direction in 3D space. The effect of fringe fields can be taken into account. Measured or externally calculated fields can be used in the simulation.

\section{SCATTERING IN GPT}

Specifically for the FEM project, scattering of electron trajectories at 3D-boundaries was introduced in GPT. It makes use of a user-defined scattering model as function of material. A combination of 3D ray-tracing techniques and Monte-Carlo statistics is used to accurately model the scattered primary particles inside the collector.

\subsection{Monte-Carlo approach}

When a macro-particle trajectory intersects a surface during the simulation, the intersection point, energy and angle of incidence are calculated. Then, the incident particle is removed from the simulation and a new macroparticle is emitted. The deflection angle and reflection energy are chosen randomly, but following a prescribed function depending on the material of the boundary [5]. The overall scattering probability is accounted for by varying the number of electrons which the new scattered macro-particle represents.

\subsection{Charge and energy dissipation}

The total charge dissipated per incident macro-particle is given by:

$Q_{\text {in }}-Q_{\text {out }}=q\left(N_{\text {in }}-N_{\text {out }}\right)$

where $N_{\text {in }}$ and $N_{\text {out }}$ represent the number of incoming and outgoing electrons which the macro-particles represent. If all incident electrons have the same energy, there is a close to linear correlation between dissipated charge and dissipated energy. However, GPT uses the general relativistic equation to be able to investigate non-linear effects:

$E_{\text {in }}-E_{\text {out }}=N_{\text {in }}\left(\gamma_{\text {in }}-1\right) m c^{2}-N_{\text {out }}\left(\gamma_{\text {out }}-1\right) m c^{2}$

where $\gamma$ is the Lorentz factor of the electrons.

\subsection{Analysis}

When a particle hits a surface, the dissipated charge and energy is recorded together with the intersection point. To ease the interpretation of the results, the 3D coordinates are "unrolled" along the collector plate. The first 
coordinate is the distance along the collector plate boundary, starting from an arbitrary point. For the second FEM collector plate, this point is marked " $\mathrm{A}$ " in Figure 2. The second coordinate is the azimuthal angle. After this transformation, inverse mapping in ray-tracing terms, the results can be presented as $2 \mathrm{D}$-density plots, a standard feature of GPTwin.

\section{SIMULATION RESULTS}

Using the scattering model of GPT, we have looked in detail at the effect of the deflection system on the current and power dissipation. Furthermore the amount of current flowing back from the collector towards the cathode was studied.

To illustrate the effect of the bending system, we show results for the second electrode of the collector. This plate receives the most current. Figure 4 shows typical GPT output for the power dissipation. The B, C, D and E markers correspond to the positions indicated in Figure 2. Without the bending system the electrons are distributed homogeneously over the plate, while with the deflection system a strong angle dependence can be seen.

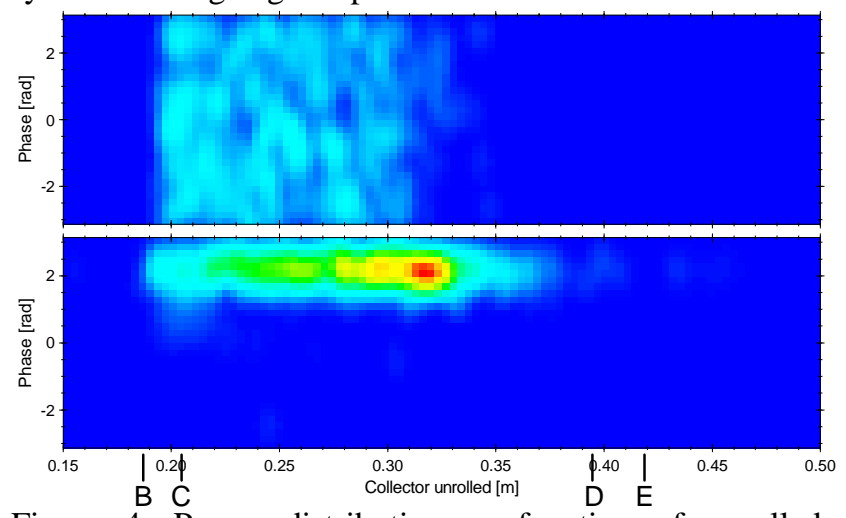

Figure 4: Power distribution as function of unrolled position and phase on second collector plate without (top) and with $1.5 \mathrm{mT}$ bending field (bottom).

Because the FEM deflection system rotates, the angle dependence is smoothed. This results in averaging the current and power dissipation over the phase along the unrolled collector coordinate. Figure 5 shows the corresponding GPT output for the second collector plate. Without the bending system there is a nearly uniform distribution over the surface of the electrode. With the deflection system the beam is bent into the collector and the electrons hit the plate at a larger radius. This information is important for the design of the cooling system, which will be installed to further increase the pulse-length. Although the local intensity peak is very high with the bending system on, when the peak is smoothed over all angles the maximum value is not higher than with the uniform angle dependency.

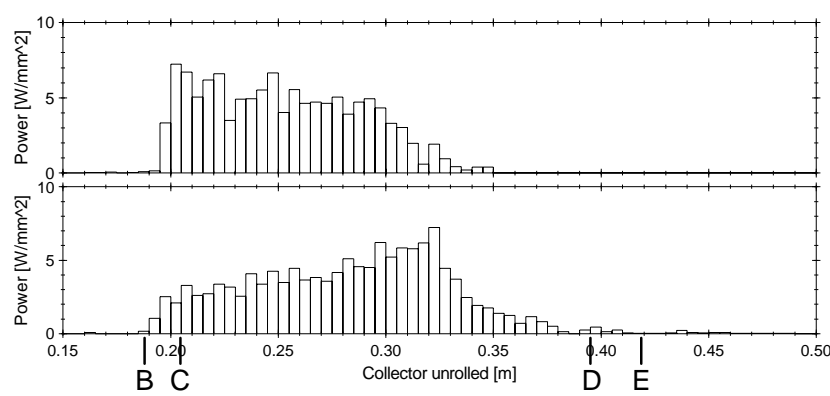

Figure 5: Power distribution as function of unrolled collector position without (top) and with $1.5 \mathrm{mT}$ bending field (bottom).

The most important effect of the collector bending system is that it lowers the current flowing back from the collector towards the cathode. As shown in Figure 6, the deflection system decreases the return current from $100 \mathrm{~mA}$ to below a tolerable $10 \mathrm{~mA}$. Therefore the deflection system is necessary to achieve the design efficiency.

The total power dissipation, summed over all collector plates, also decreases. Because the beam is actively bent into the collector, more particles hit the second plate that would otherwise have been collected on the first plate. This slightly decreases the total dissipated power at the cost of an asymmetric current load on the collector plates.

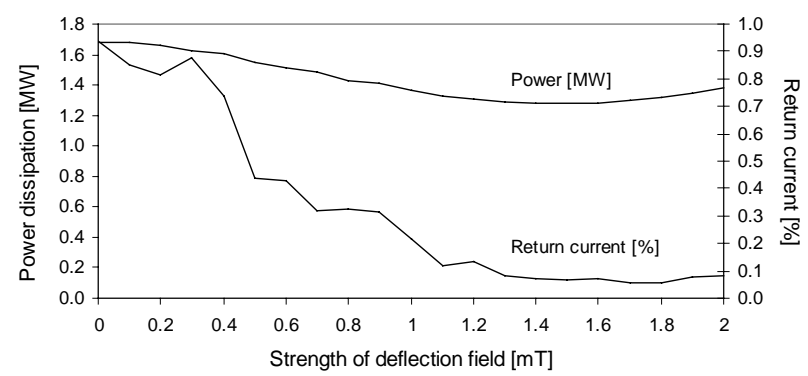

Figure 6: Total power dissipation and return current as function of bend strength.

\section{CONCLUSION}

The new scattering model in the GPT code has been used to investigate the effects of the off-axis deflection system for the FEM depressed collector. The return current is reduced to within the design specifications and the total dissipated power is reduced without affecting the maximum power dissipation on the collector plates.

\section{REFERENCES}

[1] W.H. Urbanus, et al, Nucl. Instr. and Meth. A375, (1996) pp. 401.

[2] M.J. de Loos, S.B. van der Geer, Nucl. Instr. and Meth. in Phys. Res. B, Vol. 139, (1997) pp. 481.

[3] S.B. van der Geer, M.J. de Loos, Proc. 1998 Particle Accel. Conf., Stockholm, Sweden (1998) pp. 1245.

[4] GPT User Manual, Pulsar Physics, De Bongerd 23, 3762 XA Soest, The Netherlands, http://www.pulsar.nl/gpt.

[5] J.L.H. Jonker, Philips Res. Rep. 12 (1957) pp. 249. 